\title{
VISITATION FREQUENCIES OF BUMBLEBEES AND SWALLOWTAIL BUTTERFLIES TO FLOWERS AND THE NECTAR SUGAR CONCENTRATION OF RHODODENDRON KAEMPFERI AND R. JAPONICUM IN MOUNTAINS OF CENTRAL JAPAN
}

\author{
Keigo Takahashi ${ }^{1}$ and Takao Itino*2, 3 \\ ${ }^{1}$ Graduate School of Science and Technology, Shinshu University, Matsumoto, Nagano 390-8621, Japan \\ ${ }^{2}$ Department of Biology, Faculty of Science, Shinshu University, Matsumoto, Nagano 390-8621, Japan \\ ${ }^{3}$ Institute of Mountain Science, Shinshu University, Matsumoto, Nagano 390-8621, Japan
}

\begin{abstract}
Relationships between flower visitor composition and floral traits help us to understand floral evolution. Swallowtail butterflies have been assumed to be the main pollinators of Rhododendron kaempferi and $R$. japonicum based on their floral shapes, and $R$. kaempferi was reported to be pollinated by butterflies in southern Japan. In the mountains of central and northern Japan, however, bumblebees are abundant and candidate pollinators of Rhododendron. We found that visitation frequencies of bumblebees were higher than those of swallowtail butterflies to both of the Rhododendron species at three study sites in the mountains of central Japan. $R$. japonicum pollen adhered to the bodies of both floral visitors. Further, the nectar sugar concentration of $R$. kaempferi was in the preference range of bumblebees, whereas that of $R$. japonicum was suited to both bumblebees and butterflies. Taken together, these findings support the hypothesis that bumblebees are potentially also important pollinators of $R$. kaempferi and $R$. japonicum in mountains of central Japan, where they are more abundant than swallowtail butterflies.
\end{abstract}

Keywords: bumblebee, floral characteristics, nectar, rhododendron, swallowtail butterfly

\section{INTRODUCTION}

Relationships between flower visitor composition and floral traits can help us to understand floral evolution (Kevan \& Baker 1983; Schemske \& Bradshaw I999). Suites of floral traits have evolved in response to natural selection imposed by different pollinator taxa, and these relationships are referred to as "pollination syndromes" (Johnson \& Steiner 2000; Fenster et al. 2004). In recent years, the validity of the traditional pollination syndrome concept on a world scale has been challenged (Ollerton et al. 2009; Rosas-Guerrero et al. 20I4). The expected pollinator of some plant species on the basis of their floral traits (shape, colour, scent, size, etc.) is often not realised (Ollerton et al. 2009; Vlašánková et al. 2017). In these cases, although floral traits may have initially evolved in response to the most effective pollinator (RosasGuerrero et al. 20I4), pollinator transitions can occur (Ollerton et al. 2009), which can result in floral traits that do not predict the current pollinator.

Rhododendron species can be pollinated by various animals and thus exhibit a diversity of floral traits and a range of pollination syndromes. Rhododendron calendulaceum (subgenus Pentanthera, section Pentanthera), which has big orange petals, a long pistil, and long stamens,

Received 2 March 2017, accepted I4 November 2017

${ }^{*}$ Corresponding author: itinot@shinshu-u.ac.jp is butterfly-pollinated (Epps et al. 2015). R. floccigerum (subgenus Hymenanthes, section Pontica), which has red petals with very short pistil and stamens exertion, is bumblebee- and bird-pollinated (Georgian et al. 20I5). On the other hand, $R$, aureum (subgenus Hymenanthes, section Pontica), which has medium-sized yellow flowers with relatively short pistil and stamens (Kudo I993; Kudo et al. 20II), $R$. ponticum (subgenus Hymenanthes, section Pontica), which has medium-sized purple flowers (corolla diameter 3.5 to $5 \mathrm{~cm}$ ) (Stout et al. 2006), and $R$. ferrugineum (subgenus Rhododendron, section Rhododendron), which has medium-sized pink flowers with relatively short pistil and stamens (Escaravage \& Wagner 2004), are all bumblebee-pollinated.

The shrubs R. kaempferi (subgenus Azaleastrum, section Tsutsusi) and R. japonicum (subgenus Pentanthera, section Pentanthera) have medium to large red or red-orange flowers (corolla diameter 4 and $7 \mathrm{~cm}$, respectively), and a long pistil and long stamens that stick out of the corolla. Rhododendron flowers with this colour and shape have been thought to be pollinated by large butterflies, because butterflies easily recognize reddish colours and when they alight on the corolla, their ventral abdomen is suitably positioned to brush against the long pistil and stamens (Tanaka 1993, 1997, 2001). Indeed, in the Kirishima Mountains in southern Japan, R. kaempferi is frequently visited by the swallowtail butterflies Byasa alcinous and Papilio xuthus (Yokogawa \& Hotta 1995). However, 
swallowtail butterflies are rare in northern and mountainous regions in Japan, whereas bumblebees, the other potential Rhododendron pollinator, are abundant. When the body sizes of bumblebees match the size of Rhododendron flowers, their ventral abdomen touches the anthers and the stigma (Mejias et al. 2002). Whether R. kaempferi and $R$. japonicum are pollinated by large swallowtail butterflies or by bumblebees in these central mountainous regions of Japan remains unknown.

In this paper, we pose three hypotheses. First, we hypothesize that bumblebees, not swallowtail butterflies, are main flower visitors of $R$. kaempferi and $R$. japonicum in the mountains of central Japan. Second, sugar concentration of nectar of $R$. kaempferi and $R$. japonicum is high (suited to bumblebee pollination). Third, that due to similar selection pressures by the same pollinators, flower size of $R$. kaempferi and $R$. japonicum does not vary between populations.

\section{MATERIALS AND METHODS}

\section{Study sites}

This study was carried out in Nagano Prefecture, central Japan, for 26 days from 29 April to I0 July 20I6. This period includes the peak flowering season of both Rhododendron kaempferi and $R$. japonicum. We investigated $R$. kaempferi at sites in Ikeda (Ike, $36^{\circ} 23^{\prime} \mathrm{N}$, I37 $53^{\prime} \mathrm{E} ; 633 \mathrm{~m}$ a.s.l.) and Norikura (Nor, $36^{\circ} 07^{\prime} \mathrm{N}$, I37 $37^{\circ} \mathrm{E} ; \mathrm{I}, 488 \mathrm{~m}$ a.s.1.), and $R$. japonicum at sites in Nor and Utsukushigahara (Uts, $36^{\circ} \mathrm{I} 4^{\prime} \mathrm{N}, 138^{\circ} 06^{\prime} \mathrm{E} ; \mathrm{I}, 892 \mathrm{~m}$ a.s.1.). In Ike, 50 R. kaempferi plants grew within an area of $50 \mathrm{~m} \times 50 \mathrm{~m}$ at the edge of a mixed forest in which Pinus densiflora was the dominant tree species. In Nor, a few dozen $R$. kaempferi plants grew sporadically over a distance of about I km along the edge of a mixed forest in which Quercus crispula and Malus sieboldii were dominant tree species. Also in Nor, 200-300 R. japonicum plants grew sporadically along the forest edge. In Uts, 500-600 $R$. japonicum plants grew in pastureland within an area of about $200 \mathrm{~m} \times 800 \mathrm{~m}$. We haphazardly selected I5-20 R. kaempferi and $R$. japonicum plants at each study site for the following investigation.

\section{Visitation frequencies and pollen adhesion on flower-visiting insects}

Visitation frequencies of insects, calculated as per hour per flower, were counted for a total of I0 hours for the two plant species between 08:00 and 16:00 local time (LT) at each site. We regarded an insect as a flower visitor if it inserted its mouthpart into at least one flower nectar tube in a patch. The number of $R$. kaempferi flowers in a patch was I37.8 \pm 94.6 (mean \pm S.D., $N=$ I0) in Ike and $87.8 \pm 5.5$ $(N=4)$ in Nor whilst the number of $R$. japonicum flowers in a patch was $396.8 \pm 985.4(N=8)$ in Nor and 53.4 \pm $42.8(N=7)$ in Uts. The visitation frequencies of Bombus species and Papilionidae species were compared by using a paired $t$-test on log-transformed values of visitation per flower per hour. Additionally, we observed the flowervisiting behaviour of Papilio maackii and Bombus diversus at a $R$. japonicum patch with 3,200 flowers in Nor for one hour. We recorded the time spent by insect individuals at the patch and the number of flowers visited per foraging bout.

To quantify the number of $R$. japonicum pollen grains that adhered to the bodies of the visiting insects, we placed round seals $(8 \mathrm{~mm}$ in diameter, adhesive on one side, NICHIBAN ML-I5I) on the ventral abdomen of $P$. maackii $(N=\mathrm{I})$ and on the dorsal and lateral thorax and the ventral abdomen (i.e., three seals per individual) of $B$. diversus queens $(N=4)$ in Nor on I5 June 2016. We then counted the number of pollen grains adhering to the seals under a stereoscopic microscope (Nikon SMZI500). In $R$. kaempferi, we captured a queen and a worker of $B$. ardens, and an individual of $P$. maackii in Ike and confirmed pollen grains of $R$. kaempferi attached to their bodies with the naked eye.

Although we could not test pollination efficiency directly (e.g., by single visit experiments, or other ways of measuring pollen deposition), we collected visitation data and floral characteristics data (describe below) as proxies.

\section{Daytime and night-time nectar collection quantities}

To determine whether $R$. kaempferi produced more nectar in the daytime or at night, we used $0.5 \mu \mathrm{L}$ capillary tubes (Drummond Scientific) to remove all the standing crop of nectar from 12 haphazardly selected $R$. kaempferi flowers in Nor at 16:00 LT on 3I May and then covered each flower with a nylon net to prevent insect access. The next morning, at 07:30 LT, we measured the nectar accumulated during the previous night in nine flowers (three flowers fell off the plant during the night). Then we recovered each of the nine flowers with a nylon net and, at I6:00 LT, measured the nectar volume in six flowers (three flowers fell off the plant during the day) accumulated in the daytime. We compared night-time and daytime nectar collection quantities by the Wilcoxon rank sum test ( $R$ version 3.4.0; $R$ core team 2017).

\section{Sugar concentration of nectar}

We haphazardly selected 3-20 flowers (each flower from a different plant) per site per species and measured the sugar concentration of the standing crop of nectar at 08:00 LT (three flowers of R. kaempferi in Ike, three flowers of $R$. kaempferi in Nor, three flowers of $R$. japonicum in Nor, and 20 flowers of $R$. japonicum in Uts) by using $2 \mu \mathrm{L}$ capillary tubes (Drummond Scientific) and hand-held refractometer (Bellingham and Stanley). To investigate the pattern of the large variation of sugar concentration in $R$. japonicum in Uts, we increased the sample size there. In addition, we haphazardly selected three flowers from each of four randomly selected $R$. japonicum plants in Uts and measured the sugar concentration of the standing crop of nectar between 09:00 and 12:00 LT, and between-plant differences in nectar sugar concentration were tested by using one-way ANOVA ( $R$ version 3.4.0; $R$ core team 2017).

\section{Floral size}

We haphazardly selected 20 flowers (one from each of 20 plants) per site per plant species (but I5 R. kaempferi 

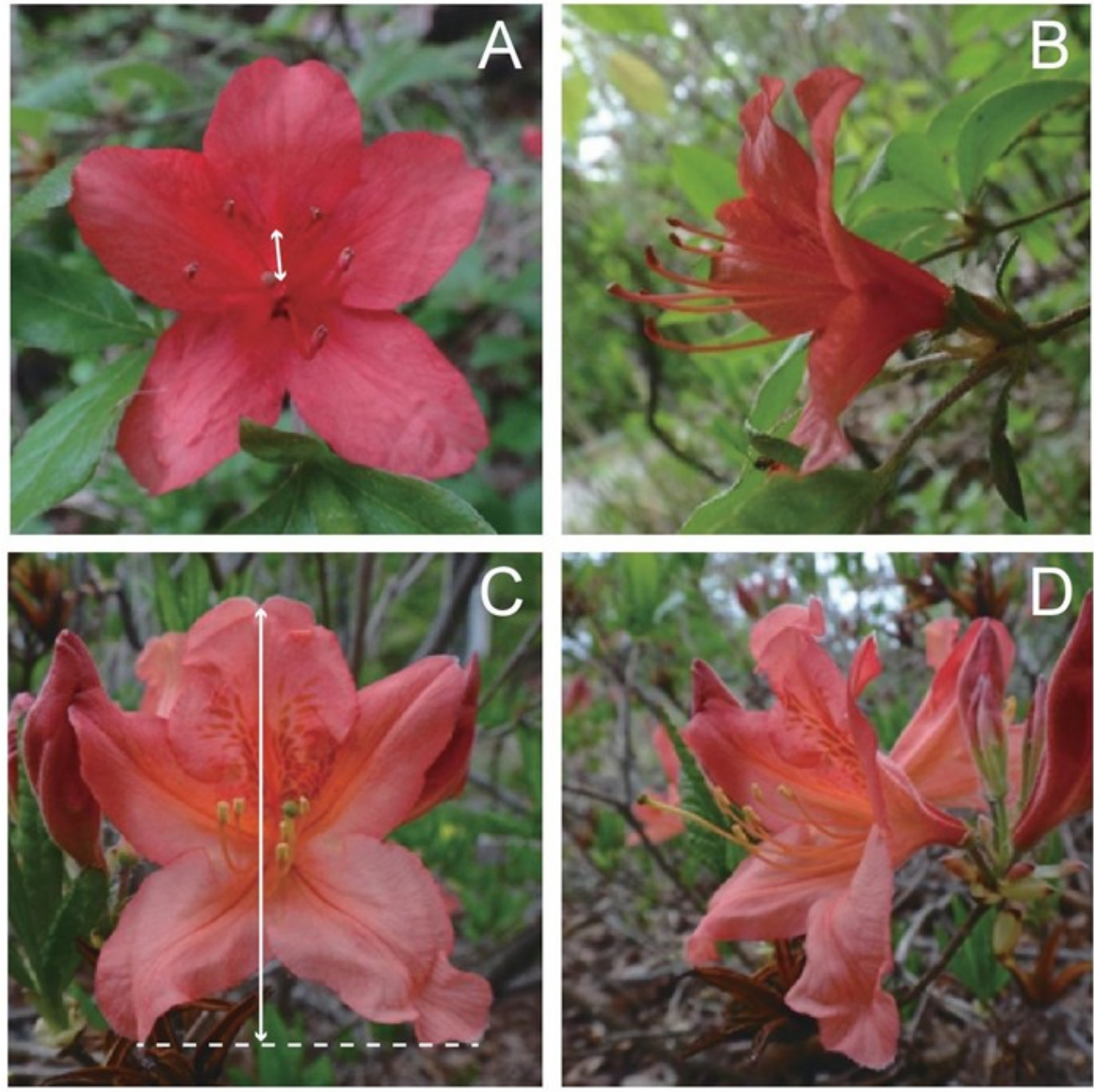

FIGURE I. Flowers of Rhododendron kaempferi (A \& $\mathrm{B})$ and $R$. japonicum (C \& D): (A) Front and (B) side view of a flower of $R$. kaempferi. In (A), there is a reddish nectar guide and a nectar tube in the position indicated by the white line. In (B), the pistil and five stamens can be seen to stick out of the corolla and curve upward. (C) Front and (D) side view of a flower of $R$. japonicum. In (C), the solid white line shows how we measured corolla diameter. The dashed line connects the tips of the two lower petals. flowers in Nor), and measured corolla diameter, lengths of the shortest and longest stamen (among five stamens), style length, and nectar tube length. Corolla diameter was defined as the distance between the top centre of the upper petal and the midpoint between the apices of the two lower petals (Fig. IC). Nectar tube refers to the tubular structure at the base of the upper petal (Fig. IA). Intraspecific and between-site differences in floral sizes were tested by using an unpaired two-tailed $t$-test.

\section{RESULTS}

Visitation frequencies and pollen adhesion on flower-visiting insects

Two species of butterfly were observed to visit Rhododendron kaempferi in Ike (Parnassius glacialis and Papilio maackii), whilst none were observed to visit $R$. kaempferi in Nor. P. maackii was also observed to visit $R$. japonicum in Nor, whilst no butterflies were observed to visit $R$. japonicum in Uts (Fig. 2). Three species of bumblebee were observed: $R$. kaempferi was visited by Bombus ardens in Ike and $B$. diversus in Nor; and $R$. japonicum was visited by $B$. ardens, B. hypocrita and $B$. diversus in Nor and B. hypocrita in Uts.

Bumblebees were more frequent visitors to $R$. kaempferi and $R$. japonicum than swallowtail butterflies at all study sites although the difference was only statistically significant for $R$. japonicum in Nor (Fig. 2).
Our limited observations of 3,200 flowers over I hour suggested that bumblebees stayed longer in a flower patch and visited more flowers per hour than butterflies. One individual of $P$. maackii perched and took nectar from only two flowers within a minute whilst three $B$. diversus queens stayed for I2, I5, and I8 minutes, visiting about I0 flowers per minute.

Queens of $B$. diversus had $44.5 \pm 35.5$ (mean \pm S.E., $N$ $=4$ ) pollen grains per seal on the dorsal thorax, I60.5 \pm 67.3 on the lateral thorax, and $125.5 \pm 47.8$ on the ventral abdomen. The swallowtail $P$. maackii had $857(N=\mathrm{I})$ pollen grains per seal on its ventral abdomen (Fig. 3 ).

\section{night}

Nectar collection quantities during the day and

In Nor, a total of $0.005 \pm 0.004 \mu \mathrm{L}$ (mean \pm S.E., $N=$ 9) of R. kaempferi nectar was extracted after flowers had been bagged overnight (for $\mathrm{I} 5$ and a half hours), and a total of $0.289 \pm 0.049 \mu \mathrm{L}(N=6)$ at the end of the following day ( 8 and a half hours, $W=0, P<0.00 I)$.

\section{Sugar concentration of nectar}

The sugar concentration of $R$. kaempferi nectar was 54.7 $\pm 4.9 \%$ (mean \pm S.E., $N=3$ ) in Ike and $5 \mathrm{I} .3 \pm 0.3 \%(N$ $=3$ ) in Nor, whereas that of $R$. japonicum nectar was 45.7 $\pm 0.7 \%(N=3)$ in Nor and $30.2 \pm 1.9 \%(N=20)$ in Uts. In Uts there was a significant difference in nectar 


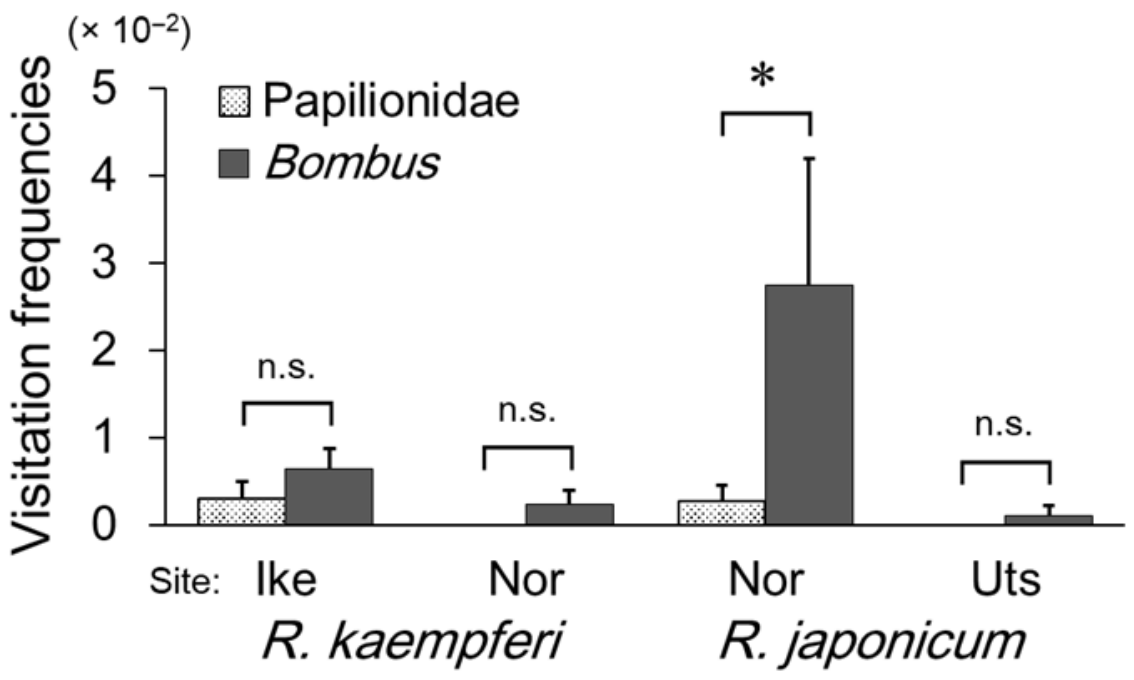

Figure 2. Visitation frequencies (number of visits per hour per flower) of Papilionidae spp. and Bombus spp. to Rhododendron kaempferi and $R$. japonicum.

${ }^{*} P<0.05$, paired $t$-test $(\log$-transformed data)

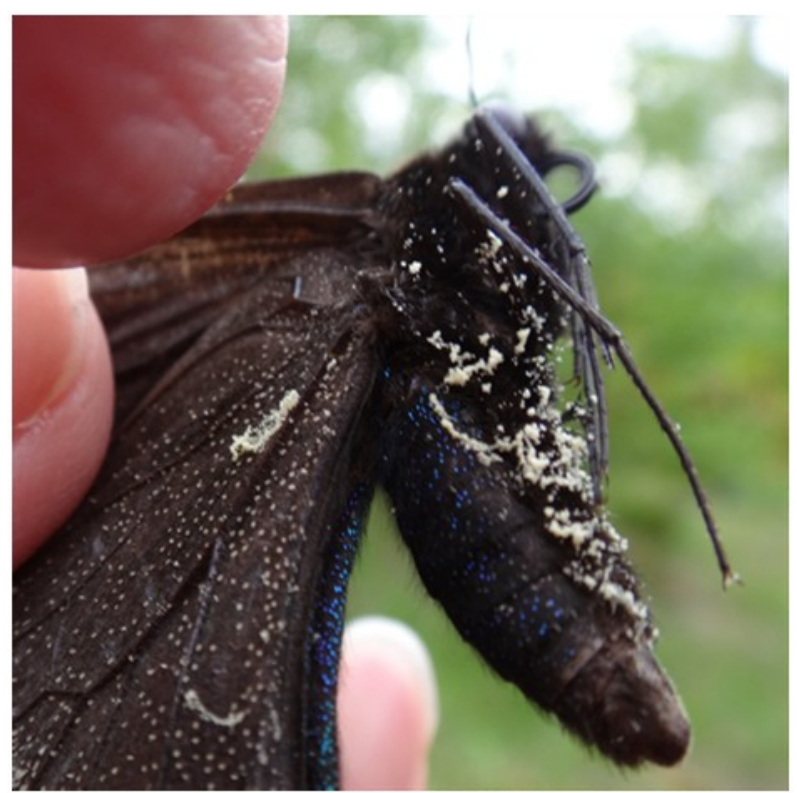

Figure 3. A Papilio maackii butterfly that has just visited a Rhododendron japonicum flower. Pollen grains are adhering to its abdomen and wings.

concentration between the four plants tested (mean \pm S.E. in Plant A: $23.3 \pm 4.1 \%$, B: $17.0 \pm 3.5 \%$, C: $22.0 \pm 1.0 \%$, and D: $33.7 \pm \mathrm{I} .2 \%$; d.f. $=3, F=6.27, P<0.05)$.

\section{Floral size}

Regional differences in floral size were not detected in $R$. kaempferi (corolla diameter: $t=0.69, P=0.49$; minimum stamen length: $t=0.32, P=0.75$; maximum stamen length: $t=0.46, P=0.65$; style length: $t=1.10, P=0.28$; nectar tube length: $t=1.26, P=0.22$; Tab. I), but detected in $R$. japonicum: flowers were larger in Nor than in Uts in terms of corolla diameter $(t=2.78, P<0.01)$, maximum stamen length $(t=2.46, P<0.05)$, and style length $(t=4.38, P<$ $0.000 \mathrm{I}$; Tab. I).

\section{DISCUSSION}

Bumblebees were more frequent visitors than swallowtail butterflies to Rhododendron kaempferi and $R$. japonicum spp., although the difference is only significant in one of four cases. Yokogawa and Hotta (I995) reported that the most frequent flower visitor to $R$. kaempferi was the swallowtail butterfly Byasa alcinous in the Kirishima Mountains, Kagoshima Prefecture $\left(31^{\circ} 53^{\prime} \mathrm{N}\right)$, southern Japan. The species diversity and abundance of swallowtail butterflies are higher in southern Japan (Kiritani 2006), whereas those of bumblebees are higher in northern and mountainous central Japan (Goulson 2003). Therefore, the main pollinator of $R$. kaempferi may change between mountainous central Japan and southern Japan (over a distance of $777 \mathrm{~km}$ ) according to local bee and butterfly diversity and abundance.

Although Tanaka (1993, I997, 200I) inferred that floral shape of Rhododendron spp. is adapted to swallowtail butterfly pollination in Japan, pollen grains also adhere to the bodies of the flower-visiting bumblebees, suggesting that they could also be pollinators of $R$. kaempferi and $R$. japonicum. When bumblebees hovered in front of the corolla, they changed their position frequently and pollen grains adhered all parts of their bodies. On the other hand, when the swallowtail butterfly $P$. maackii visited a flower, it landed on the pistil and stamens and sucked nectar with its legs and the ventral side of abdomen being in contact with the stigma and anthers, which extend outward horizontally with slightly curving tips in both $R$. kaempferi and $R$. japonicum. This more precise pollen placement may have implications for the relative efficiency of butterflies vs. bumblebees as pollinators, but this requires further investigation.

The adaptation of nectar production time has been reported to be a component of a pollination syndrome (Cruden \& Hermann-Parker 1979; Itino et al. I99I). $R$. kaempferi nectar was mainly produced in the daytime, making it suited to daytime pollinators such as swallowtail 
TABLE I. Sizes (mean \pm S.E.; $\mathrm{mm}$ ) of floral parts of Rhododendron kaempferi and $R$. japonicum. $N=$ number of flowers measured per species per site. Tests were conducted to compare sizes between the two populations of each species.

\begin{tabular}{|c|c|c|c|c|c|c|}
\hline \multirow{2}{*}{$\begin{array}{l}\text { Species } \\
\text { Site }\end{array}$} & \multicolumn{3}{|c|}{ Rhododendron kaempferi } & \multicolumn{3}{|c|}{ Rhododendron japonicum } \\
\hline & Ike & Nor & & Nor & Uts & \\
\hline Altitude (m) & 633 & $\mathrm{I} 439-\mathrm{I} 480$ & & $\mathrm{I} 440-\mathrm{I} 488$ & $1829-1892$ & \\
\hline$N$ & 20 & 15 & & 20 & 20 & \\
\hline Corolla diameter & $42.3 \pm 0.8$ & $43 . \mathrm{I} \pm \mathrm{I} .0$ & n.s. & $69.5 \pm 1.9$ & $62.3 \pm \mathrm{I} .7$ & 㚳 \\
\hline Stamen length (Min) & $31.3 \pm 0.6$ & $30.9 \pm 0.8$ & n.s. & $4 \mathrm{I} .5 \pm \mathrm{I} . \mathrm{I}$ & $39.3 \pm 0.9$ & n.s. \\
\hline Stamen length (Max) & $36.5 \pm 0.6$ & $37.0 \pm 1.0$ & n.s. & $47.8 \pm 1.2$ & $44.2 \pm 0.8$ & \# \\
\hline Style length & $36.5 \pm 0.7$ & $37.9 \pm \mathrm{I} .2$ & n.s. & $53.3 \pm 1.2$ & $46.4 \pm \mathrm{I} .0$ & 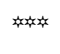 \\
\hline Nectar tube length & $12.9 \pm 0.4$ & $\mathrm{I} 3.7 \pm 0.5$ & n.s. & $\mathrm{I} 4.8 \pm 0.5$ & $\mathrm{I} 3.7 \pm 0.4$ & n.s. \\
\hline
\end{tabular}

${ }^{*} P<0.05 ; P<0.01 ; P<0.0001 ;$ n.s.: not significant, unpaired two-tailed $t$-test.

butterflies and bumblebees, although it would have been better to add a treatment reversing the order between daytime collection and night-time collection to test production in day vs. night independently. We do not know if the same is true for $R$. japonicum and this would be worth further exploration.

The mean nectar sugar concentration of $R$. kaempferi (5I-54\%) and $R$. japonicum (30-45\%) suggests an adaptation to pollination by either bumblebees, which prefer nectar with a high sugar concentration (30-55\%, Willmer 20II), or butterflies (butterfly-pollinated flowers sugar concentration ranges between 17-40\%, Willmer 20II). However, in Uts, the nectar sugar concentration of $R$. japonicum varied from $19 \%$ to $45 \%$ between individual plants. Chwil and Weryszko-Chmielewska (2009) also reported that, in Poland, the sugar concentration of $R$. japonicum nectar rises ontogenetically from $19 \%$ (on the third day of flowering) to $37 \%$ (on the fourth day of flowering). Since our sample sizes were small, and did not take into account flower age, the patterns of variation in the nectar sugar concentration warrant a future research.

Nagano et al. (20I4) and Kuriya et al. (2015) showed that flower size and pollinator size were correlated in Campanula punctata and Prunella vulgaris, respectively, across regions several kilometres apart, reflecting adaptation of flowers to the pollinators distributing each region with different elevations. In contrast to these plant species that are adapted to bumblebee pollination, we expected that flower size of $R$. kaempferi and $R$. japonicum that are visited not only by bumblebees but also by swallowtail butterflies does not vary between populations. As a result, it is not surprising that we found no differences in floral size between sites for $R$. kaempferi. Smaller flowers of $R$. japonicum at one site may just have been a response to local abiotic conditions.

We conclude that both bumblebees and swallowtail butterflies are potential pollinators of $R$. kaempferi and $R$. japonicum in the study sites. Further investigation is required to determine the real effective pollinator (bumblebees or butterflies) of $R$. kaempferi and $R$. japonicum, and to see whether the pollinator-related traits of $R$. kaempferi and $R$. japonicum changes between populations depending on the abundance of and adaptation to the effective pollinator group.

\section{ACKNOWLEDGEMENTS}

We thank the Chubu District Forest Office (Forestry Agency) and the Matsumoto Regional Office (Nagano Prefectural Government) for permission to work in the study areas. This research was funded by the Japan Ministry of Education, Culture, Sports, Science and Technology (I5H0264I to TI).

\section{REFERENCES}

Chwil M, Weryszko-Chmielewska E (2009) Characteristics of nectaries and nectar in flowers of two Rhododendron species. Journal of Apicultural Science 53: 17-27.

Cruden RW, Hermann-Parker SM (1979) Butterfly pollination of Caesalpinia pulcherrima, with observations on a psychophilous syndrome. The Journal of Ecology 67: 155-168.

Epps MJ, Allison SE, Wolfe LM (2015) Reproduction in flame azalea (Rhododendron calendulaceum, Ericaceae): A rare case of insect wing Pollination. The American Naturalist I86: 294-30I.

Escaravage N, Wagner J (2004) Pollination effectiveness and pollen dispersal in a Rhododendron ferrugineum (Ericaceae) population. Plant Biology 6: 606-6I5.

Fenster CB, Armbruster WS, Wilson P, Dudash MR, Thomson JD (2004) Pollination syndromes and floral specialization. Annual Review of Ecology, Evolution, and Systematics: 375-403.

Georgian E, Fang Z, Emshwiller E, Pidgeon A (2015) The pollination ecology of Rhododendron floccigerum Franchet (Ericaceae) in Weixi, Yunnan Province, China. Journal of Pollination Ecology I6: 72-8I.

Goulson D (2003) Bumblebees: their behaviour and ecology. Oxford University Press, New York.

Itino T, Kato M, Hotta M (I99I) Pollination ecology of the two wild bananas, Musa acuminata subsp. halabanensis and $M$ salaccensis: chiropterophily and ornithophily. Biotropica 23: I5II58.

Johnson SD, Steiner KE (2000) Generalization versus specialization in plant pollination systems. Trends in Ecology \& Evolution I5: I40-I43. 
Kevan PG, Baker HG (1983) Insects as flower visitors and pollinators. Annual Review of Entomology 28: 407-453.

Kiritani K (2006) Predicting impacts of global warming on population dynamics and distribution of arthropods in Japan. Population Ecology 48: 5-I2.

Kudo G (I993) Relationship between flowering time and fruit set of the entomophilous alpine shrub, Rhododendron aureum (Ericaceae), inhabiting snow patches. American Journal of Botany 80: I300-I304.

Kudo G, Hirao AS, Kawai Y (20II) Pollination efficiency of bumblebee queens and workers in the alpine shrub Rhododendron aureum. International Journal of Plant Sciences I72: 70-77.

Kuriya S, Hattori M, Nagano Y, Itino T (2015) Altitudinal flower size variation correlates with local pollinator size in a bumblebeepollinated herb, Prunella vulgaris L. (Lamiaceae). Journal of Evolutionary Biology 28: 1761-1769.

Mejias JA, Arroyo J, Ojeda F (2002) Reproductive ecology of Rhododendron ponticum (Ericaceae) in relict Mediterranean populations. Botanical Journal of the Linnean Society I40: 2973II.

Nagano Y, Abe K, Kitazawa T, Hattori M, Hirao AS, Itino T (2014) Changes in pollinator fauna affect altitudinal variation of floral size in a bumblebee-pollinated herb. Ecology and Evolution 4: 3395-3407.

Ollerton J, Alarcón R, Waser NM, Price MV, Watts S, Cranmer L, Hingston A, Peter CI, Rotenberry J (2009) A global test of the pollination syndrome hypothesis. Annals of Botany I03: I47II480.
R Core Team (2017) R: A language and environment for statistical computing. R Foundation for Statistical Computing, Vienna, Austria. URL https://www.R-project.org/.

Rosas-Guerrero V, Aguilar R, Martén-Rodríguez S, Ashworth L, Lopezaraiza-Mikel M, Bastida JM, Quesada M (20I4) A quantitative review of pollination syndromes: do floral traits predict effective pollinators? Ecology Letters I7: 388-400.

Schemske DW, Bradshaw HD (1999) Pollinator preference and the evolution of floral traits in monkeyflowers (Mimulus). Proceedings of the National Academy of Sciences 96: II9I0II9I5.

Stout JC, Parnell JA, Arroyo J, Crowe TP (2006) Pollination ecology and seed production of Rhododendron ponticum in native and exotic habitats. Biodiversity \& Conservation I5: 755777.

Tanaka H (1993) Introduction to floral ecology. Nouson Bunkasha, Tokyo. (In Japanese)

Tanaka H (1997) Ecology of flower-insect interactions. Hoikusha, Osaka. (In Japanese)

Tanaka H (200I) Mutual deception between flowers and insects. Kodansha, Tokyo. (In Japanese)

Vlašánková A, Padyšáková E, Bartoš $\mathrm{M}$, Mengual X, Janečková $\mathrm{P}$, Janeček $\check{S}$ (2017) The nectar spur is not only a simple specialization for long-proboscid pollinators. New Phytologist 2I5: I574-I58I.

Willmer P (20II) Pollination and floral ecology. Princeton University Press, Princeton.

Yokogawa M, Hotta M (1995) Taxonomical notes on plants of southern Japan II. Variation in the Rhododendron obtusum group in the Kirishima Mts., southern Kyushu. Acta Phytotaxonomica et Geobotanica 46: I65-I83. 